\title{
Repetitive Transcranial Magnetic
} Stimulation for Improving Cognitive Function in Patients With Mild Cognitive Impairment: A Systematic
Review

\author{
Lijuan Jiang ${ }^{1 \dagger}$, Huiru Cui ${ }^{1+}$, Caidi Zhang ${ }^{1 \dagger}$, Xinyi Cao ${ }^{1}$, Nannan Gu ${ }^{1}$, Yikang Zhu ${ }^{1}$, \\ Jijun Wang ${ }^{1,2,3}$, Zhi Yang ${ }^{1,2,4,5}$ and Chunbo $L^{1,2,3,4 *}$
}

\begin{abstract}
${ }^{1}$ Shanghai Key Laboratory of Psychotic Disorders, Shanghai Mental Health Center, Shanghai Jiao Tong University School of Medicine, Shanghai, China, ${ }^{2}$ Institute of Psychology and Behavioral Science, Shanghai Jiao Tong University, Shanghai, China, ${ }^{3}$ Center for Excellence in Brain Science and Intelligence Technology (CEBSIT), Chinese Academy of Science, Beijing, China, ${ }^{4}$ Brain Science and Technology Research Center, Shanghai Jiao Tong University, Shanghai, China, ${ }^{5}$ Laboratory of Psychological Heath and Imaging, Shanghai Mental Health Center, Shanghai Jiao Tong University School of Medicine, Shanghai, China
\end{abstract}

Background: Mild cognitive impairment (MCl) is an early stage of Alzheimer's disease. Repetitive transcranial magnetic stimulation (rTMS) has been widely employed in $\mathrm{MCl}$ research. However, there is no reliable systematic evidence regarding the effects of rTMS

OPEN ACCESS

Edited by:

Dennis Qing Wang,

Southern Medical University, China

Reviewed by:

Xie Fen,

Southern Medical University, China Csaba Jozsef Nyakas,

Semme/weis University, Hungary

*Correspondence:

Chunbo L

licb@smhc.org.cn

tThese authors have contributed equally to this work and share first authorship

Received: 09 August 2020 Accepted: 02 December 2020 Published: 14 January 2021

Citation:

Jiang L, Cui H, Zhang C, Cao X, Gu N,

Zhu Y, Wang J, Yang Z and Li C

(2021) Repetitive Transcranial Magnetic Stimulation for Improving Cognitive Function in Patients With

Mild Cognitive Impairment: A Systematic Review.

Front. Aging Neurosci. 12:593000

doi: 10.3389/fnagi.2020.593000 on $\mathrm{MCl}$. The aim of this review was to evaluate the efficacy and safety of rTMS in the treatment of $\mathrm{MCl}$.

Methods: A comprehensive literature search of nine electronic databases was performed to identify articles published in English or Chinese before June 20, 2019. The identified articles were screened, data were extracted, and the methodological quality of the included trials was assessed. The meta-analysis was performed using the RevMan 5.3 software. We used the GRADE approach to rate the quality of the evidence.

Results: Nine studies comprising 369 patients were included. The meta-analysis showed that rTMS may significantly improve global cognitive function (standardized mean difference [SMD] 2.09, 95\% confidence interval [Cl] 0.94 to 3.24, $p=0.0004$, seven studies, $n=296$; low-quality evidence) and memory (SMD 0.44, 95\% Cl 0.16 to $0.72, p=0.002$, six studies, $n=204$; moderate-quality evidence). However, there was no significant improvement in executive function and attention $(p>0.05)$. Subgroup analyses revealed the following: (1) rTMS targeting the left hemisphere significantly enhanced global cognitive function, while rTMS targeting the bilateral hemispheres significantly enhanced global cognitive function and memory; (2) high-frequency rTMS significantly enhanced global cognitive function and memory; and (3) a high number of treatments $\geq 20$ times could improve global cognitive function and memory. There was no significant difference in dropout rate $(p>0.05)$ between the rTMS and control groups. However, patients who received rTMS had a higher rate of mild adverse effects (risk ratio 2.03, $95 \% \mathrm{Cl} 1.16$ to 3.52, $p=0.01$, seven studies, $n=317$; moderate-quality evidence).

Conclusions: rTMS appears to improve global cognitive function and memory in patients with $\mathrm{MCl}$ and may have good acceptability and mild adverse effects. 
Nevertheless, these results should be interpreted cautiously due to the relatively small number of trials, particularly for low-frequency rTMS.

Keywords: repetitive transcranial magnetic stimulation, mild cognitive impairment, cognitive function, systematic review, meta-analysis

\section{INTRODUCTION}

Mild cognitive impairment (MCI) is the transitional stage between normal aging and dementia, in which individuals have subjective cognitive deficits and objective memory impairment without impairments in daily activities (Petersen et al., 1999). Along with cognitive decline, MCI implies an increased risk of falls, slow walking speed, and physical frailty (LeWitt et al., 2020; $\mathrm{Ma}$ and Chan, 2020). The mechanisms underlying cognitive decline are multifactorial, including inflammation, impaired hypothalamic-pituitary axis stress response, imbalanced energy metabolism, mitochondrial dysfunction, oxidative stress, and endocrine dysfunction (Zou et al., 2018; Xu et al., 2019a; Li et al., 2020; Ma and Chan, 2020; Wang et al., 2020a). The annual conversion rate of $\mathrm{MCI}$ to dementia ranges from 10 to $15 \%$, demonstrating that it is an important condition to identify and treat (Petersen et al., 2009; Ding et al., 2016). Existing pharmacological interventions for MCI are unsatisfactory and have limited effectiveness (Sanford, 2017). Therefore, nonpharmacological interventions for MCI have received increasing attention (Feng et al., 2018; Kasper et al., 2020).

Recently, the use of non-invasive brain stimulation has garnered considerable clinical and research interest (Hsu et al., 2015; Xu et al., 2019b). Repetitive transcranial magnetic stimulation (rTMS) is a non-invasive method of brain stimulation in which a train of magnetic pulses is delivered to a specific target location of the brain (Wei et al., 2017). rTMS involves trains of magnetic pulses of various frequencies and intensities. As a general rule, high frequencies $(\geq 5 \mathrm{~Hz})$ increase cortical excitability and low frequencies $(\leq 1 \mathrm{~Hz})$ suppress it (Lin et al., 2019). rTMS has been widely studied in patients with various neuropsychiatric illnesses such as depression, epilepsy, Parkinson's disease, and schizophrenia (Najib et al., 2011; Guo et al., 2017).

In recent years, several meta-analyses have investigated the effects of rTMS in older patients with cognitive impairment, demonstrating that rTMS may have a beneficial effect on cognitive function (Hsu et al., 2015; Wang et al., 2015, 2020b; Cheng et al., 2018; Dong et al., 2018; Lin et al., 2019; Chou et al., 2020). Four reviews focused on patients with Alzheimer's disease (AD) (Hsu et al., 2015; Dong et al., 2018; Lin et al., 2019; Wang et al., 2020b), and two reviews included MCI and AD patients, but did not analyze MCI patients separately (Cheng et al., 2018; Chou et al., 2020). Moreover, only one review in China comprising small sample sizes has investigated the effects of rTMS in patients with MCI (Wang et al., 2015). Despite the growing body of evidence supporting the beneficial effects of rTMS in older patients with cognitive impairment, the relationships between the effect of rTMS and factors such as target site, parameter settings, and treatment course still require further investigation.
Therefore, the aim of this systematic review and meta-analysis was to provide up-to-date evidence on the effects of rTMS on cognitive function in MCI patients.

\section{MATERIALS AND METHODS}

This work adhered to the Preferred Reporting Items for Systematic Reviews and Meta-Analyses (PRISMA) guidelines (Liberati et al., 2009) and was registered in the open access database International Prospective Register of Systematic Reviews (PROSPERO) (http://www.crd.york.ac.uk/PROSPERO/; registration number: CRD42019126269).

\section{Search Strategies}

The following databases were searched to identify studies on the effect of rTMS on MCI, published before June 20, 2019: PubMed, ISI Web of Science, Embase, the Cochrane Library, EBSCO, Chinese National Knowledge Infrastructure (CNKI), Chinese Technical Periodicals (VIP), Wanfang Database, and China BioMedical Literature database (SinoMed). The English keywords used for the database searches were "mild cognitive impairment," "MCI," "transcranial magnetic stimulation," "repetitive transcranial magnetic stimulation," "TMS," and "rTMS." The Chinese keywords were "Qingdurenzhizhangai," "Qingdurenzhisunhai," "Chongfujingluciciji." The reference lists of identified articles were checked for other potential studies.

\section{Inclusion and Exclusion Criteria}

To be included, studies had to meet the following criteria: (1) they were randomized controlled studies investigating the effects of rTMS treatment on the cognitive function of patients with MCI; (2) they included participants diagnosed with MCI based on any diagnostic criteria, such as the Petersen criteria, the National Institute on Aging-Alzheimer's Association criteria for MCI due to AD (Albert et al., 2011), or the fifth edition of the Diagnostic and Statistical Manual of Mental Disorders; (3) the experimental group received rTMS, regardless of stimulation site and stimulation frequency. rTMS could be combined with other interventions such as drug therapy or cognitive training; (4) the control group received sham rTMS stimulation, medication, or other interventions; (5) only the first treatment period of cross-over trials was considered; (6) outcomes included global cognitive ability and specific domain of cognition, which were measured by neuropsychological tests or other objective measurements.

Studies were excluded if (a) they were animal studies; (b) included participants with vascular cognitive impairment or other neurological disorders resulting from dementia or Parkinson's disease; (c) they were reviews, conference presentations, or unpublished reports; (d) they were duplicate 
reports; (e) they used a blank control as their control group; or (f) they just had a single TMS pulse intervention or studies with $\leq 1$ week of intervention time.

\section{Evaluation of the Quality of Studies}

We evaluated the quality of included studies based on the criteria specified in the Cochrane Handbook for Systematic Reviews of Interventions (Higgins and Green, 2011) and the Grading of Recommendations, Assessment, Development, and Evaluation (GRADE) framework (Guyatt et al., 2008). Two researchers (LJ and CZ) independently extracted data from each included study and compared their results. When discrepancies occurred, they discussed their differences and a consensus was reached; if necessary, a third researcher was asked to resolve any remaining differences. If possible, the authors of the original article were contacted if the information was unclear or insufficient.

\section{Evaluation of Risk of Bias}

The risk of bias was assessed using the method recommended by the Cochrane Collaboration (Higgins and Green, 2011). The following characteristics were evaluated: (a) adequacy of sequence generation; (b) allocation concealment; (c) use of blinding; (d) how incomplete outcome data (dropouts) were addressed; (e) evidence of selective outcome reporting; and (f) other potential risks that may harm the validity of the study. The risk of bias for each domain was graded as low, high, or unclear.

\section{Evaluation of Quality of Evidence}

The GRADE approach (Guyatt et al., 2008) was used to categorize the quality of evidence provided in each report into four levels: (a) "high quality": further research was unlikely to affect the reliability of the efficacy evaluation results; (b) "medium quality": further research was likely to affect the reliability of the efficacy evaluation results and very likely to change the outcome of the evaluation; (c) "low quality": further research was very likely to affect the reliability of the efficacy evaluation results and the evaluation outcome was very likely to change; and (d) "very low quality": results of any efficacy evaluation were uncertain. GRADEpro software (McMaster University, Hamilton, Canada) was used to edit, analyze, and graph the level of evidence. If a randomized controlled trial was defective, the quality of the evidence was downgraded by one or two levels.

\section{Data Extraction}

A data extraction table was constructed and two researchers extracted and double-checked data from the included articles. The following information was extracted: basic study information (study authors, year of publication, study design), participant characteristics (age, and sample size), rTMS parameters [stimulus site, true stimulus frequency, stimulus intensity (\% of resting motor threshold), and treatment regimen], cognitive outcome measures, dropout rate, and adverse effects. Motor threshold (MT) was defined as the minimum stimulator intensity capable of inducing a visible muscle twitch of the contralateral hand in at least $50 \%$ of a series of ten single-pulse TMS trials (Rossini et al., 1994). Cognitive outcomes were classified into three cognitive domains:
(1) global cognitive function [e.g., the Mini-Mental State Examination (Folstein et al., 1975)]; (2) memory (encoded, stored, and retrieved of information); (3) executive function and attention (multi-dimensional and complex cognitive constructs) (Karssemeijer et al., 2017). If cognitive outcome data were reported from multiple time points, those from immediately after the intervention were obtained for meta-analysis.

\section{Data Analysis}

RevMan 5.3 statistical software (The Nordic Cochrane Center, The Cochrane Collaboration, Copenhagen, Denmark) was used to conduct statistical analyses. Quantitative variables were summarized using standardized mean differences (SMDs); qualitative variables were summarized using risk ratios (RRs). Corresponding 95\% confidence intervals (CIs) were also calculated. Pooled results were presented using forest plots. The degree of heterogeneity was evaluated using the Q statistic generated from the $\chi^{2}$ test. The degree of statistical heterogeneity was assessed by the $I^{2}$ statistic. Studies with $I^{2}<50 \%$ and $p$ $\geq 0.1$ were considered homogeneous and a fixed-effects model was used; studies with $I^{2}>50 \%$ or $p<0.1$ were considered heterogeneous and a random-effects model was used. Subgroup analyses were performed separately according to stimulus site, stimulus frequency, and treatment course. We also performed sensitivity analysis if necessary. Funnel plots were used to assess the possibility of publication bias.

\section{RESULTS}

\section{Search and Selection of Studies}

The study selection process is shown in Figure 1. A total of 629 potentially relevant studies were identified from five English and four Chinese databases using relevant search strategies. Of these relevant studies, 120 duplicates were removed, and the eligibility of the remaining 509 studies was further assessed. During the title and abstract screening phase, an additional 442 articles were removed. Finally, after the full texts of the remaining 67 articles were reviewed, 58 articles were excluded, thus nine studies were included in this meta-analysis.

\section{Characteristics of the Included Studies}

Table 1 shows the characteristics of the nine studies included in this meta-analysis, comprising a total of 369 participants (187 in the rTMS group and 182 in the control group). Two trials (Drumond Marra et al., 2015; Cui et al., 2019) were in English, and the remaining were in Chinese. Participants were diagnosed with MCI based on one of the following diagnostic criteria: the Petersen criteria, European Consortium on Alzheimer's Disease criteria, American Society of Neuroscience Quality Standards Branch criteria, National Institute on Aging-Alzheimer's Association criteria, or Chinese expert consensus on the prevention and treatment of cognitive dysfunction criteria. Only one study included low-frequency rTMS (Wan et al., 2016); all other studies used high-frequency rTMS (Han et al., 2013; Yang et al., 2014; Zhang et al., 2014; Drumond Marra et al., 2015; Sun and Ma, 2015; Long et al., 2016; Wen et al., 2018; Cui et al., 2019). rTMS stimulation sites included the left dorsolateral 


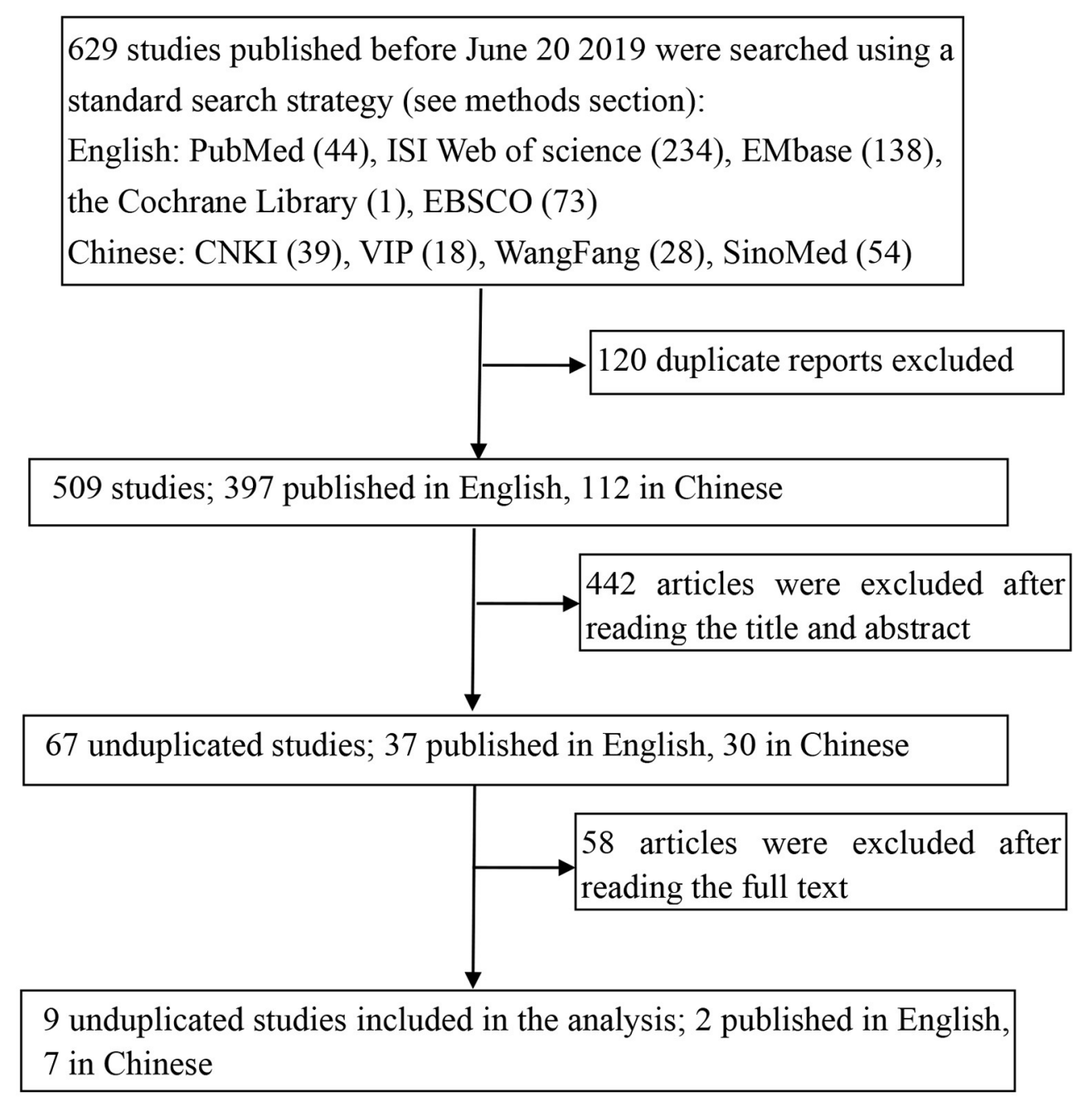

FIGURE 1 | Flowchart of the literature search and screening processes.

prefrontal cortex (DLPFC) (Drumond Marra et al., 2015; Sun and Ma, 2015; Long et al., 2016; Wen et al., 2018), right DLPFC (Cui et al., 2019), bilateral DLPFC (Han et al., 2013; Yang et al., 2014), bilateral prefrontal cortex (PFC) (Zhang et al., 2014), and bilateral anterior temporal lobe (Wan et al., 2016), with a stimulation intensity from 80 to $110 \%$ resting MT. The repetition number of intervention was 10-48 times. The interventions in the control group, which included sham rTMS stimulation [three studies used sham coil (Yang et al., 2014; Drumond Marra et al., 2015; Long et al., 2016); three studies rotated the coil 90 degrees to achieve the effect of sham therapy (Han et al., 2013; Wen et al., 2018; Cui et al., 2019)], medication treatment (Zhang et al., 2014; Wan et al., 2016), and cognitive training (Sun and Ma, 2015).

With respect to outcome measures, different cognitive measurement tools were applied to assess the same cognitive domains within a study or among studies. Measures of global cognitive function included the Montreal Cognitive Assessment (MoCA) (five studies) (Han et al., 2013; Zhang et al., 2014; Sun and Ma, 2015; Long et al., 2016; Wen et al., 2018), and Mini-Mental State Examination (MMSE) (two studies) (Yang et al., 2014; Cui et al., 2019). Memory was measured using the associative learning test (one study) (Han et al., 2013), Rivermead Behavioral Memory Test (RBMT) (two studies) (Drumond Marra et al., 2015; Wen et al., 2018), Clinical Memory Scale (CMS) (two studies) (Long et al., 2016; Wan et al., 2016), logic memory test (one study) (Cui et al., 2019). To assess executive function and attention two studies used the Trial Making Test-A (TMT-A) (Han et al., 2013; Cui et al., 2019), two studies used the Trial Making Test-B (TMT-B) (Drumond Marra et al., 2015; Cui et al., 2019), and two studies used the verbal fluency test (VFT) (Han et al., 2013; Drumond Marra et al., 2015) (Table 2).

\section{Research Quality}

The summary of the risk of bias of the included studies is shown in Figure 2. All included trials reported random allocation, but only five of the nine studies described the method used to generate the random sequence in detail and were thus rated "low risk" (Yang et al., 2014; Drumond Marra et al., 2015; Wan et al., 2016; Wen et al., 2018; Cui et al., 2019). Only one study described the allocation concealment procedure in detail 
TABLE 1 | Characteristics of the nine included studies.

\begin{tabular}{|c|c|c|c|c|c|c|c|c|}
\hline References & $\begin{array}{l}\text { Study } \\
\text { design }\end{array}$ & Interventions & Age $(M \pm S D)$ & $\begin{array}{l}\text { Sample size } \\
\text { (M/F) }\end{array}$ & $\begin{array}{l}\text { Site for } \\
\text { stimulation }\end{array}$ & $\begin{array}{l}\text { Stimulation } \\
\text { frequency } \\
\text { Stimulation } \\
\text { intensity (\%MT) }\end{array}$ & $\begin{array}{l}\text { Treatment } \\
\text { frequency, } \\
\text { Number of pulses } \\
\text { each time }\end{array}$ & $\begin{array}{l}\text { Cognitive outcomes/ } \\
\text { Measure }\end{array}$ \\
\hline Han et al., 2013 & Parallel & $\begin{array}{l}\text { T: active rTMS } \\
\text { C: sham rTMS }\end{array}$ & $\begin{array}{l}66.5(5.02) \\
66.7(5.25)\end{array}$ & $\begin{array}{l}22(8 / 14) \\
18(6 / 12)\end{array}$ & $\begin{array}{l}\text { Bilateral } \\
\text { DLPFC }\end{array}$ & $\begin{array}{l}20 \mathrm{~Hz} \\
80 \%\end{array}$ & $\begin{array}{l}40 \text { times } \\
36,000 \text { pulses }\end{array}$ & $\begin{array}{l}\text { Global cognitive } \\
\text { function: MoCA; } \\
\text { Memory: associative } \\
\text { learning test, episodic } \\
\text { memory test; Executive } \\
\text { function and attention: } \\
\text { TMT-A, WCST, VFT, } \\
\text { DSST }\end{array}$ \\
\hline Yang et al., 2014 & Parallel & $\begin{array}{l}\text { T: active rTMS } \\
\text { C: sham rTMS }\end{array}$ & $\begin{array}{l}66(6) \\
66(7)\end{array}$ & $\begin{array}{l}18(8 / 10) \\
15(7 / 8)\end{array}$ & $\begin{array}{l}\text { Bilateral } \\
\text { DLPFC }\end{array}$ & $\begin{array}{l}20 \mathrm{~Hz} \\
80 \%\end{array}$ & $\begin{array}{l}40 \text { times } \\
36,000 \text { pulses }\end{array}$ & $\begin{array}{l}\text { Global cognitive } \\
\text { function: MMSE }\end{array}$ \\
\hline $\begin{array}{l}\text { Zhang et al., } \\
2014\end{array}$ & Parallel & $\begin{array}{l}\text { T: active rTMS } \\
\mathrm{C} \text { : } \\
\text { piracetam treatment }\end{array}$ & $\begin{array}{l}65.6(8.9) \\
65.9(9.9)\end{array}$ & $\begin{array}{l}25(12 / 13) \\
25(13 / 12)\end{array}$ & Bilateral PFC & $\begin{array}{l}5 \mathrm{~Hz} \\
100 \%\end{array}$ & $\begin{array}{l}24 \text { times } \\
800 \text { pulses }\end{array}$ & $\begin{array}{l}\text { Global cognitive } \\
\text { function: MoCA }\end{array}$ \\
\hline $\begin{array}{l}\text { Sun and Ma, } \\
2015\end{array}$ & Parallel & $\begin{array}{l}\text { T: active rTMS + } \\
\text { cognitive training } \\
\text { C: cognitive training }\end{array}$ & $\begin{array}{l}65.4(5.6) \\
63.4(8.2)\end{array}$ & $\begin{array}{l}40(23 / 17) \\
40(20 / 20)\end{array}$ & $\begin{array}{l}\text { Left DLPFC } \\
\text { and left PFC }\end{array}$ & $\begin{array}{l}15 \mathrm{~Hz} \\
80-110 \%\end{array}$ & $\begin{array}{l}48 \text { times } \\
\text { NA }\end{array}$ & $\begin{array}{l}\text { Global cognitive } \\
\text { function: MoCA }\end{array}$ \\
\hline Long et al., 2016 & Parallel & $\begin{array}{l}\text { T: active rTMS } \\
\text { C: sham rTMS }\end{array}$ & $\begin{array}{l}68.27(9.85) \\
65.63(9.36)\end{array}$ & $\begin{array}{l}15(8 / 7) \\
15(6 / 9)\end{array}$ & Left DLPFC & $\begin{array}{l}15 \mathrm{~Hz} \\
90 \%\end{array}$ & $\begin{array}{l}10 \text { times } \\
1,000 \text { pulses }\end{array}$ & $\begin{array}{l}\text { Global cognitive } \\
\text { function: MoCA; } \\
\text { Memory: CMS }\end{array}$ \\
\hline Wan et al., 2016 & Parallel & $\begin{array}{l}\text { T: active rTMS + } \\
\text { conventional drug } \\
\text { treatment } \\
\text { C: conventional } \\
\text { drug treatment }\end{array}$ & $\begin{array}{l}65.44(9.61) \\
65.11(5.39)\end{array}$ & $\begin{array}{l}18(12 / 6) \\
18(11 / 7)\end{array}$ & $\begin{array}{l}\text { Bilateral } \\
\text { Anterior temporal }\end{array}$ & $\begin{array}{l}1 \mathrm{~Hz} \\
80 \%\end{array}$ & $\begin{array}{l}15 \text { times } \\
600 \text { pulses }\end{array}$ & Memory: CMS \\
\hline
\end{tabular}

ACE-III, Addenbrooke's Cognitive Examination-III; AVLT, auditory verbal learning test; CMS, Clinical Memory Scale; DLPFC, Dorsolateral Prefrontal Cortex; DSST, Digit Symbol Substitution Test; F, female; M, male; M, mean; MT, Motor Threshold; MMSE, Mini-Mental State Examination; MoCA, Montreal Cognitive Assessment; NA, no data or not describe; RBMT, Rivermead Behavioral Memory Test; rTMS, repetitive Transcranial Magnetic Stimulation; SD, Standard deviation; TMT, Trial Making Tests; VFT, Verbal Fluency Test; WAIS-III, Wechsler Adult Intelligence Scale III; WCST: Wisconsin Card Sorting Test; WMS, Wechsler Memory Scale.

(Drumond Marra et al., 2015). In three studies, the participants and researchers were blinded, thus performance bias was rated as "low risk" (Yang et al., 2014; Drumond Marra et al., 2015; Cui et al., 2019). The risk of attrition bias in five studies was rated as "high risk" because the research data were incomplete (due to drop-out and adverse effects) and intention-to-treat analysis was not implemented (Han et al., 2013; Yang et al., 2014; Drumond Marra et al., 2015; Sun and Ma, 2015; Cui et al., 2019). Two studies were selective in reporting their results, thus reporting bias was rated as "high risk" (Drumond Marra et al., 2015; Cui et al., 2019). Studies with unclear information were rated as "unclear risk."

\section{Meta-Analysis of Treatment Effect Global Cognitive Function}

Seven studies with a total of 296 participants with MCI assessed the effects of rTMS on global cognitive ability. The heterogeneity of the included studies was high $\left(I^{2}=93 \%, p<0.00001\right)$, so a random-effects model was used for the meta-analysis. The funnel plot revealed significant asymmetry (Figure 3A). However, it is usually recommended that $\geq 10$ studies are required to make a definitive conclusion about publication bias, so this result may be considered suggestive of, but not definite evidence of, publication bias. Due to the high heterogeneity between studies, a sensitivity analysis was conducted. Omitting 
TABLE 2 | GRADE quality of evidence assessment of individual outcome indicators for the efficacy of repetitive transcranial magnetic stimulation in the treatment of mild cognitive impairment.

\begin{tabular}{|c|c|c|c|c|c|c|c|c|c|}
\hline \multirow[t]{2}{*}{ Outcome indicator } & \multirow{2}{*}{$\begin{array}{c}\text { Number of } \\
\text { included cases }\end{array}$} & \multicolumn{2}{|c|}{ Heterogeneity } & \multirow{2}{*}{$\begin{array}{l}\text { Model of } \\
\text { analysis }\end{array}$} & \multicolumn{2}{|c|}{ Group effect value } & \multirow[t]{2}{*}{ Estimated value } & \multirow[t]{2}{*}{$95 \% \mathrm{Cl}$} & \multirow[t]{2}{*}{ Grade } \\
\hline & & $I^{2}$ & $p$ & & $z$ & $p$ & & & \\
\hline Global cognitive function & 296 & $93 \%$ & $<0.00001$ & Random effect & 3.55 & 0.0004 & 2.09 (SMD) & $0.94,3.24$ & Low \\
\hline Memory & 204 & $26 \%$ & 0.24 & Fixed effect & 3.07 & 0.002 & 0.44 (SMD) & $0.16,0.72$ & Moderate \\
\hline $\begin{array}{l}\text { Executive function and } \\
\text { attention }\end{array}$ & 93 & $49 \%$ & 0.14 & Fixed effect & 0.92 & 0.36 & $-0.19(\mathrm{SMD})$ & $-0.61,0.22$ & Moderate \\
\hline Drop-out rate & 260 & $7 \%$ & 0.37 & Fixed effect & 0.01 & 0.99 & $0.99(\mathrm{RR})$ & $0.39,2.54$ & Moderate \\
\hline Adverse effect & 317 & $0 \%$ & 0.66 & Fixed effect & 2.50 & 0.01 & $2.03(\mathrm{RR})$ & $1.16,3.52$ & Moderate \\
\hline
\end{tabular}

$S M D$, standardized mean difference; RR, relative risk; Cl, confidence interval; GRADE, Grading of Recommendation Assessment, Development and Evaluation.

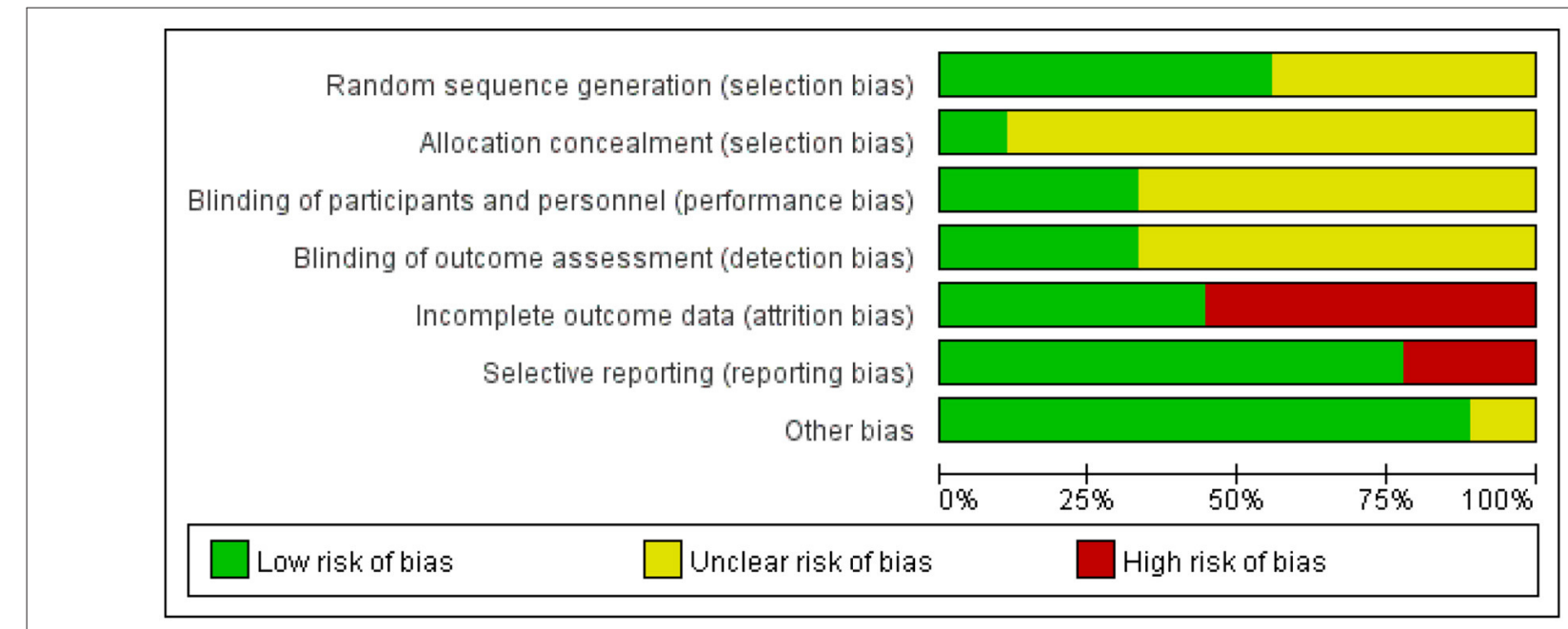

FIGURE 2 | Risk of bias graph: review authors' judgements about each risk of bias item presented as percentages across all included studies.

one study with low standard deviation (Zhang et al., 2014) did not significantly alter the pooled SMD. The combined results demonstrated that the rTMS group had significantly improved global cognitive function (SMD 2.09, 95\% CI 0.94 to $3.24, p=$ 0.0004) compared to the control group (Figure 4). According to the GRADE system, the overall level of evidence with respect to the effect of rTMS on global cognitive function was "low" (Table 2).

\section{Subgroup Analysis of Global Cognitive Function}

A subgroup analysis was conducted according to the stimulation site (the left hemisphere, right hemisphere, and bilateral hemispheres). The subgroup analysis revealed a standardized mean difference of 1.38 (95\% CI 0.24 to 2.51) for trials involving "left hemisphere" stimulation and a standardized mean difference of $-0.02 \quad(95 \%$ CI -0.88 to 0.84$)$ for trials involving "right hemisphere" stimulation. The mean effect size for trials involving "bilateral hemispheres" stimulation was 3.89 (95\% CI 0.87 to 6.91) (Figure 5).

The number of treatment sessions in the included studies ranged from 10 to 48 times. We divided the studies into two groups: those with a high number of treatments $(\geq 20$ times) and those with a low number of treatments $(<20$ times). The subgroup analysis revealed that the standardized mean difference for studies with a high number of treatments was 2.92 (95\% CI 1.43 to 4.40$)$. Studies with a low number of treatments showed a standardized mean difference of $0.24(95 \% \mathrm{CI}-0.32$ to 0.79$)$. These results indicate that a high number of rTMS treatments produced better global cognitive function than a low number of rTMS treatments (Figure 6). 

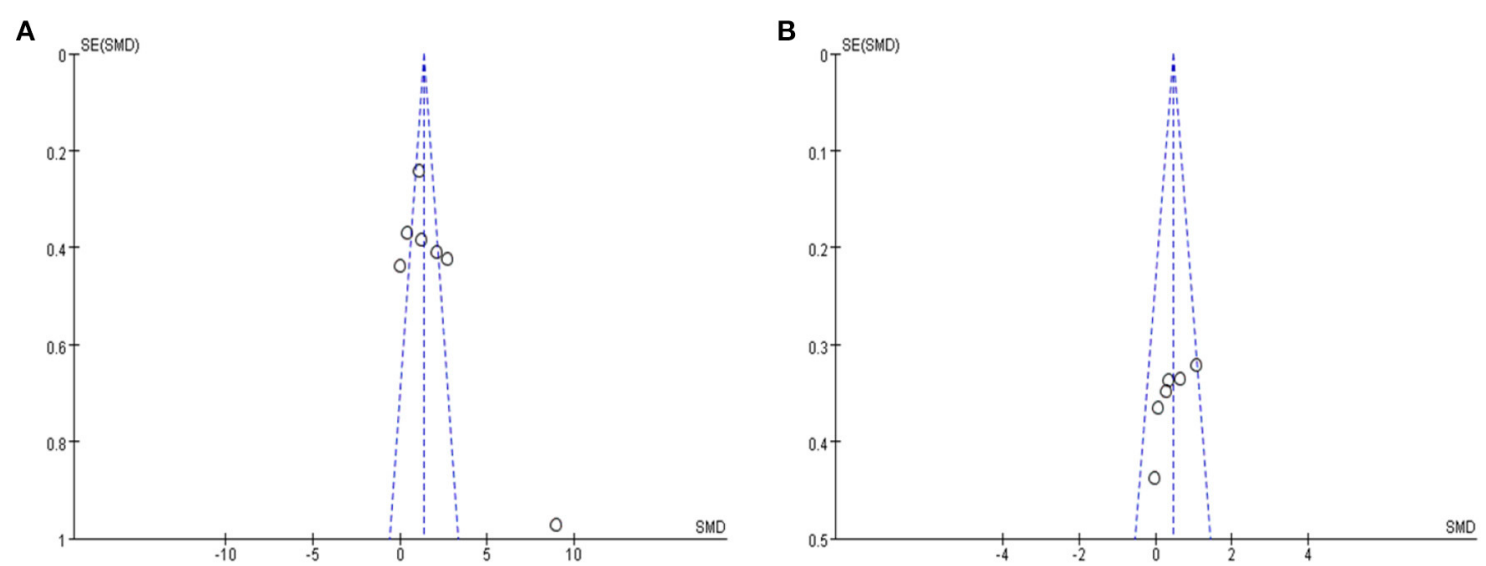

FIGURE 3 | (A) Funnel plot for the publication bias of global cognitive function (B) Funnel plot for the publication bias of memory. SMD, standardized mean difference.

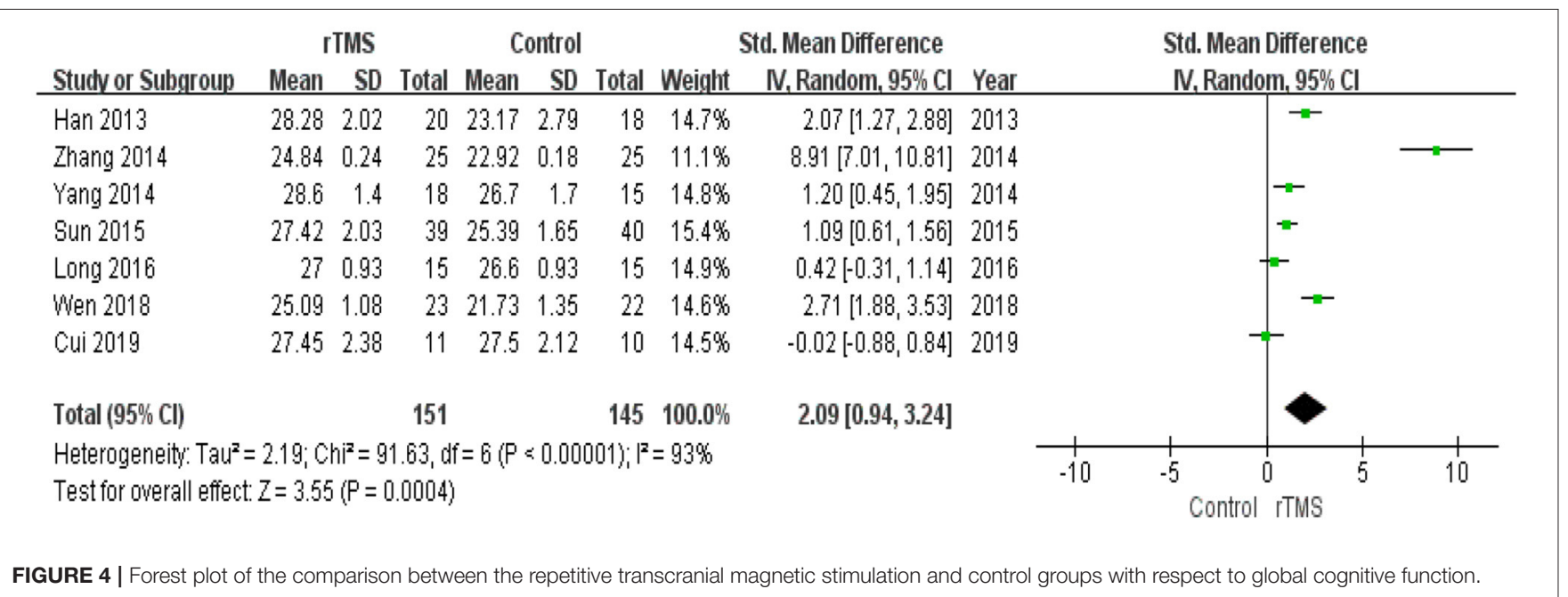

\section{Memory}

Six studies reported the effects of rTMS on memory. The heterogeneity of the included studies was low $\left(I^{2}=26 \%, p=\right.$ $0.24)$; therefore, a fixed-effects model was applied. The funnel plot did not reveal significant asymmetry (Figure 3B). The results of the meta-analysis showed that rTMS had a significant effect on memory improvement (SMD 0.44, 95\% CI 0.16 to $0.72, p=$ 0.002) compared to the control group (Figure 7). According to the GRADE system, the overall level of evidence with respect to the effect of rTMS on memory was "moderate" (Table 2).

\section{Subgroup Analysis of Memory}

A subgroup analysis was conducted according to the stimulation site (the left hemisphere, right hemisphere, and bilateral hemispheres). The subgroup analysis revealed a standardized mean difference of 0.48 ( $95 \%$ CI -0.13 to 1.09 ) for trials involving "left hemisphere" stimulation and -0.04 (95\% CI -0.90 to 0.81 ) for trials involving "right hemisphere" stimulation. The standardized mean difference for trials involving "bilateral hemispheres" stimulation was 0.49 ( $95 \%$ CI 0.02 to 0.95$)$. No significant difference in the effect size of rTMS for MCI was observed in this subgroup analysis (Figure 8).

The studies were divided into two groups according to the administered frequency of stimulation: a high-frequency stimulation group $(\geq 5 \mathrm{~Hz})$ and a low-frequency stimulation group $(\leq 1 \mathrm{~Hz})$. This subgroup analysis revealed no significant difference between the high-frequency subgroup (SMD 0.47, 95\% CI 0.16 to $0.78, p=0.003$ ) and low-frequency subgroup (SMD $0.32,95 \% \mathrm{CI}-0.33$ to $0.98, p=0.33$ ) (Figure 9).

Subgroup analysis of the number of treatment sessions showed that participants in the high number of treatments had a significant improvement $(p=0.02)$ in memory compared with the controls (Figure 10).

\section{Executive Function and Attention}

The effects of rTMS on executive function and attention were measured in three studies. The heterogeneity of the included studies was low $\left(I^{2}=49 \%, p=0.14\right)$, so a fixed-effects model was 


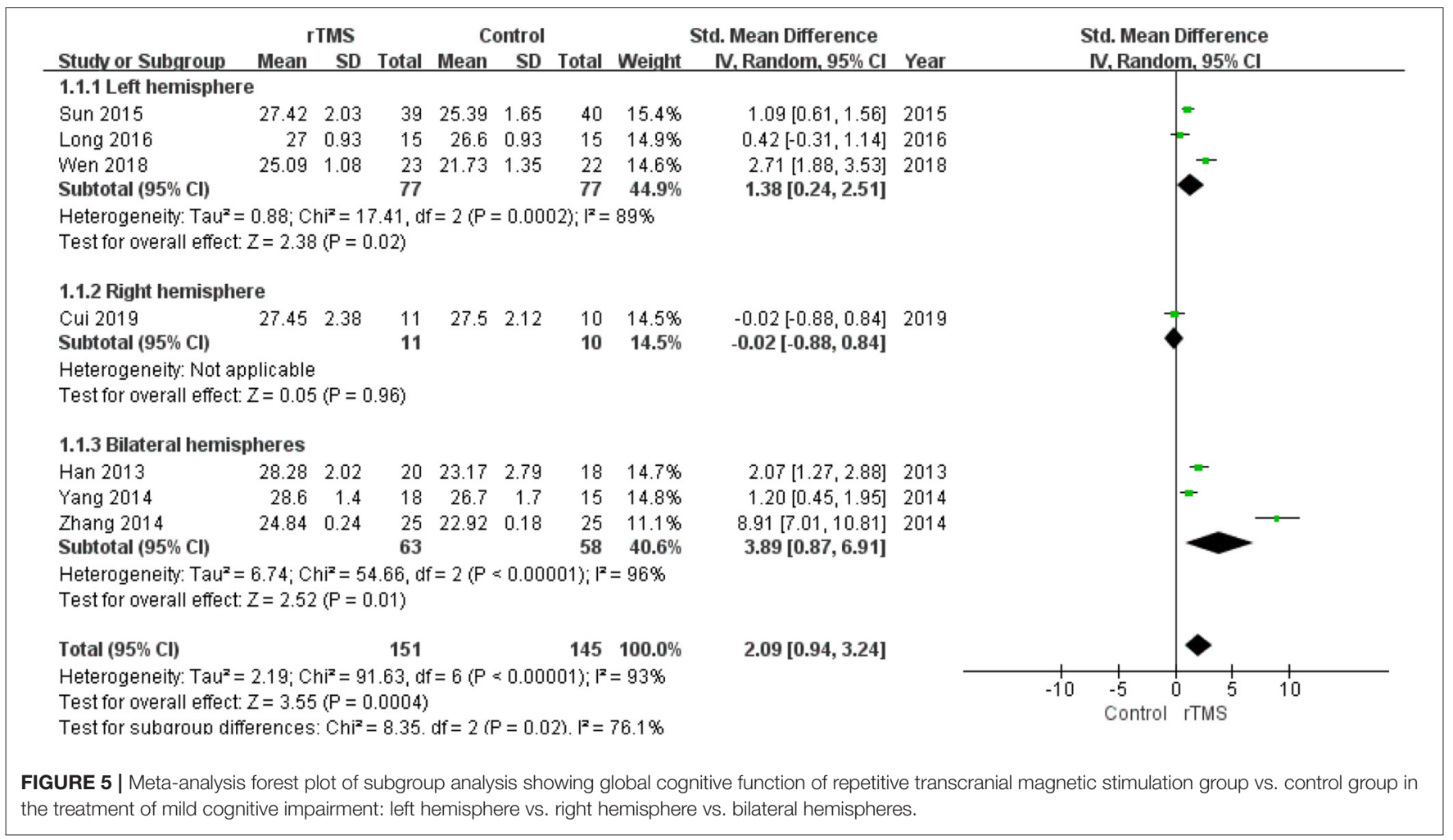

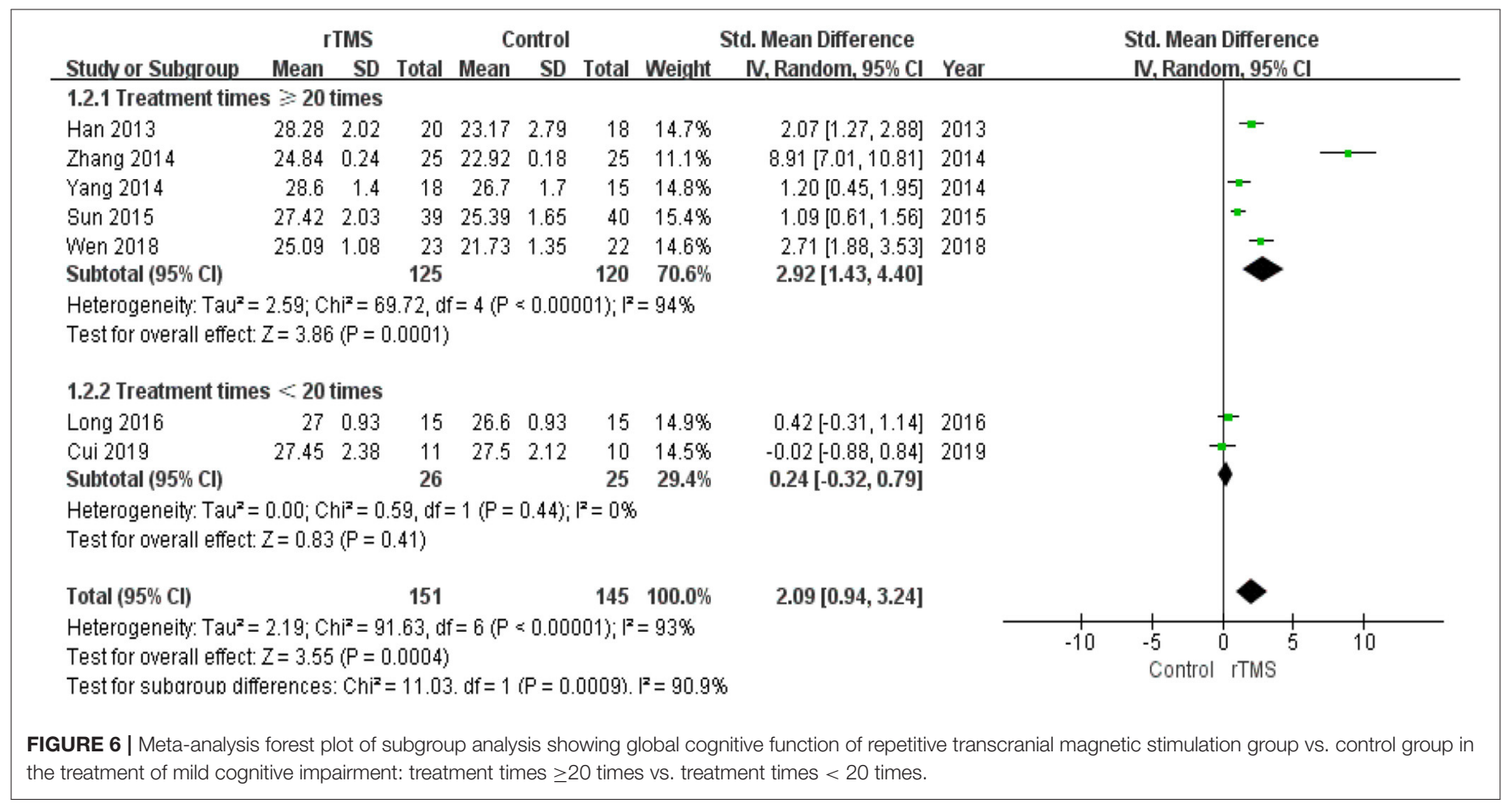

used for the meta-analysis. Meta-analysis showed no significant differences (SMD $-0.19,95 \% \mathrm{CI}-0.61$ to $0.22, p=0.36$, three studies, $n=93$; moderate-quality evidence) between the rTMS and control groups (Supplementary Figure 1).

\section{Meta-Analysis of Dropout Rate}

Six studies comprising a total sample size of 260 cases reported dropouts. Based on the results of the heterogeneity test $\left(I^{2}=\right.$ $7 \%, p=0.37)$, we used a fixed-effects model. The pooled 


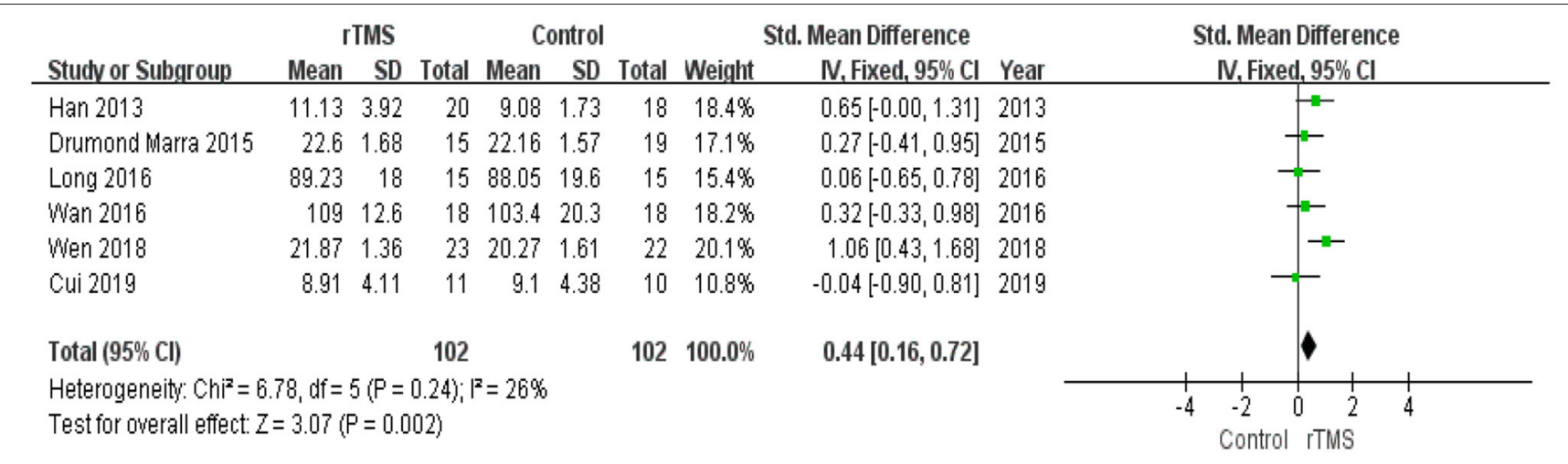

FIGURE 7 | Forest plot of the comparison between the repetitive transcranial magnetic stimulation and control groups with respect to memory.

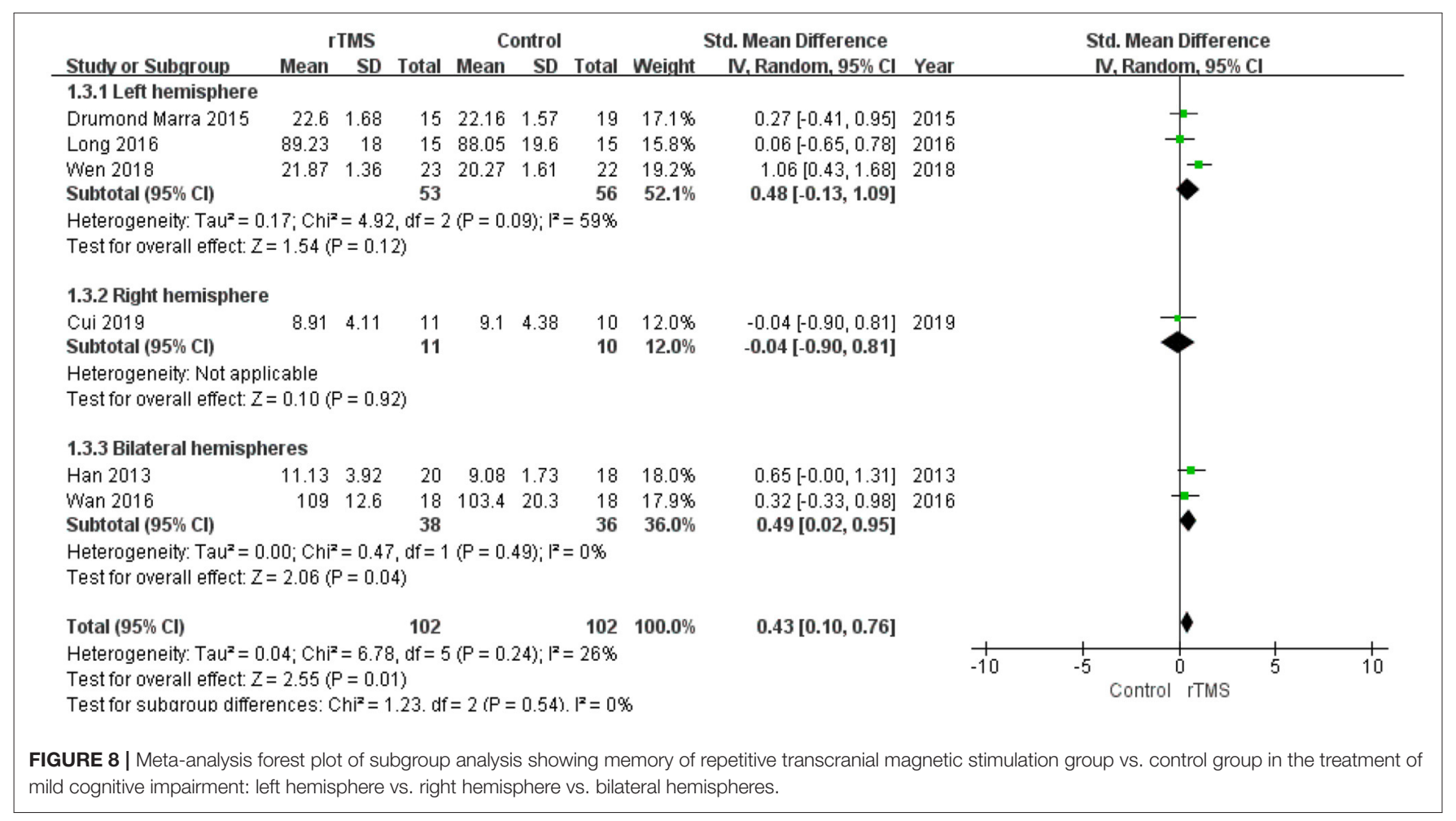

results showed no differences in dropout rate between the rTMS group and the control group (RR $0.99,95 \%$ CI 0.39 to 2.54, $p=0.99$, six studies, $n=260$; moderate-quality evidence) (Supplementary Figure 2).

\section{Meta-Analysis of Adverse Effects}

None of the included studies reported serious adverse effects. Seven studies reported mild adverse effects. Discomfort was reported by 26 of the 160 patients in the rTMS group and 12 of the 157 patients in the control group. Other adverse effects reported included headache, dizziness, pain in the stimulated area, tinnitus, cervical pain, and concentration difficulties. No heterogeneity was found among the studies $\left(I^{2}=0 \%, p=0.66\right)$. The pooled results showed that the rTMS group had a higher incidence of adverse effects than control group ( $\mathrm{RR} 2.03,95 \%$ CI 1.16 to $3.52, p=$ 0.01 , seven studies, $n=317$; moderate-quality evidence) (Supplementary Figure 3).

\section{DISCUSSION}

\section{Main Findings}

This systematic review identified nine studies comprising a total of 369 participants with MCI, of which 187 were treated with active rTMS for 10 to 48 times and 182 were treated with sham rTMS, cognitive training, or drug treatment. Overall, the results of our meta-analysis support the benefits of rTMS on global cognitive function and memory in patients with MCI. 


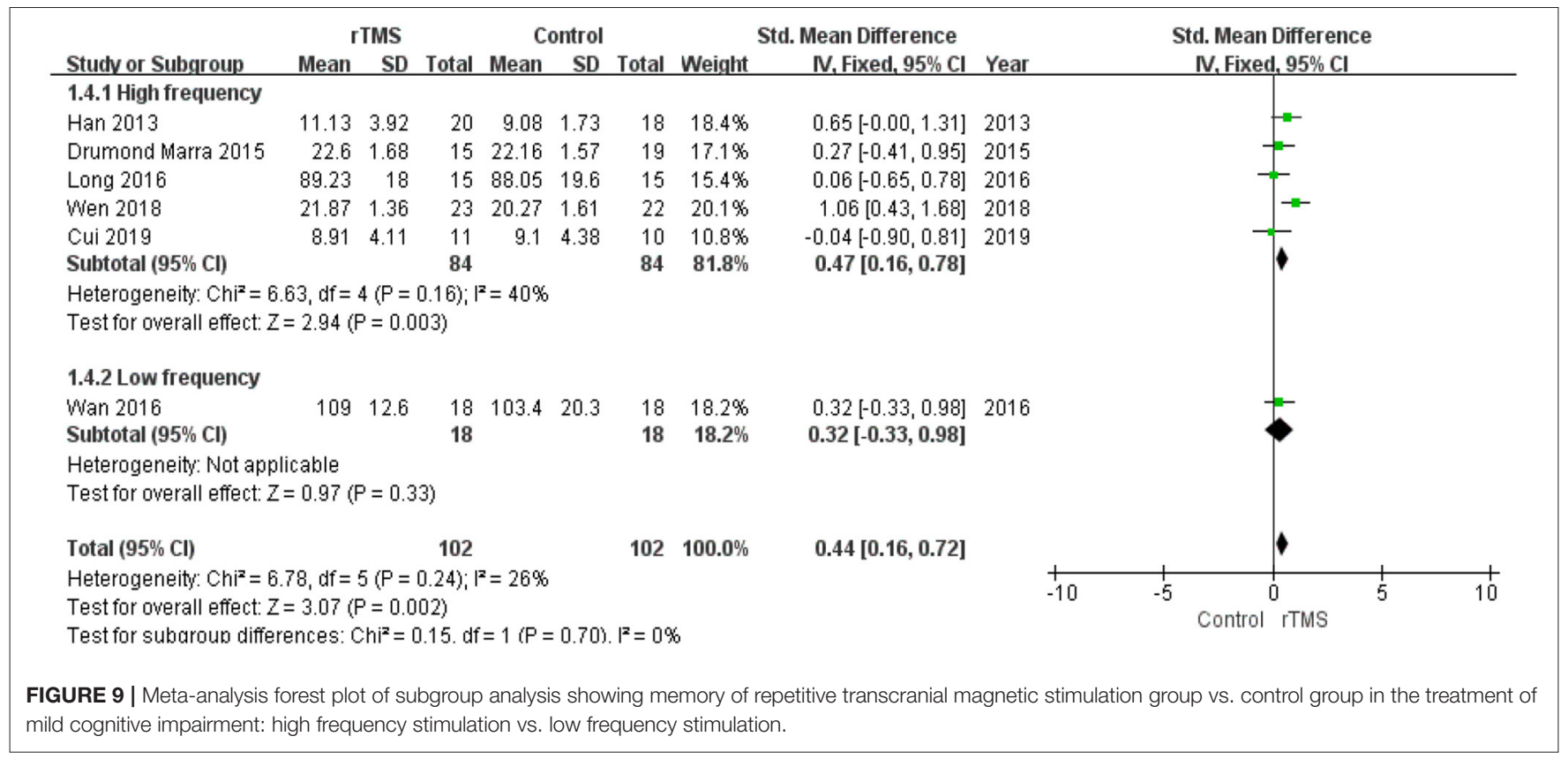

\begin{tabular}{|c|c|c|c|c|c|c|c|c|c|c|c|c|}
\hline \multirow[b]{2}{*}{ Studv or Subgroup } & \multicolumn{2}{|c|}{ rTMS } & \multicolumn{3}{|c|}{ Control } & \multicolumn{3}{|r|}{ Std. Mean Difference } & \multirow{2}{*}{\multicolumn{4}{|c|}{$\begin{array}{l}\text { Std. Mean Difference } \\
\text { IV. Random. } 95 \% \mathrm{Cl}\end{array}$}} \\
\hline & Mean & SD & Total & Mean & SD & Total & Weight & IV, Random, $95 \% \mathrm{Cl}$ & & & & \\
\hline \multicolumn{13}{|c|}{ 1.5.1 Treatment times $\geqslant 20$ times } \\
\hline Han 2013 & 11.13 & 3.92 & 20 & 9.08 & 1.73 & 18 & $18.0 \%$ & $0.65[-0.00,1.31]$ & 2013 & & $t=$ & \\
\hline Wen 2018 & 21.87 & 1.36 & 23 & 20.27 & 1.61 & 22 & $19.2 \%$ & $1.06[0.43,1.68]$ & 2018 & & - & \\
\hline Subtotal $(95 \% \mathrm{Cl})$ & & & 43 & & & 40 & $37.2 \%$ & $0.86[0.41,1.32]$ & & & $\boldsymbol{\varphi}$ & \\
\hline \multicolumn{13}{|c|}{$\begin{array}{l}\text { Heterogeneity: } \text { Tau }^{2}=0.00 ; \mathrm{Ch}^{2}=0.77, \mathrm{df}=1(\mathrm{P}=0.38) ; \mathrm{I}^{2}=0 \% \\
\text { Test for overall effect: } \mathrm{Z}=3.73(\mathrm{P}=0.0002)\end{array}$} \\
\hline \multicolumn{13}{|c|}{ 1.5.2 Treatment times $<20$ times } \\
\hline Drumond Marra 2015 & 22.6 & 1.68 & 15 & 22.16 & 1.57 & 19 & $17.1 \%$ & $0.27[-0.41,0.95]$ & 2015 & & 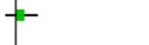 & \\
\hline Long 2016 & 89.23 & 18 & 15 & 88.05 & 19.6 & 15 & $15.8 \%$ & $0.06[-0.65,0.78]$ & 2016 & & - & \\
\hline $\operatorname{Wan} 2016$ & 109 & 12.6 & 18 & 103.4 & 20.3 & 18 & $17.9 \%$ & $0.32[-0.33,0.98]$ & 2016 & & - & \\
\hline Cui 2019 & 8.91 & 4.11 & 11 & 9.1 & 4.38 & 10 & $12.0 \%$ & $-0.04[-0.90,0.81]$ & 2019 & & - & \\
\hline Subtotal $(95 \% \mathrm{Cl})$ & & & 59 & & & 62 & $62.8 \%$ & $0.18[-0.18,0.54]$ & & & 9 & \\
\hline \multicolumn{13}{|c|}{$\begin{array}{l}\text { Heterogeneity: Tau }=0.00 ; \mathrm{Chi}^{2}=0.61, \mathrm{df}=3(\mathrm{P}=0.89) ; \mathrm{I}^{2}=0 \% \\
\text { Test for overall effect: } \mathrm{Z}=0.97(\mathrm{P}=0.33)\end{array}$} \\
\hline Total $(95 \% \mathrm{Cl})$ & & & 102 & & & 102 & $100.0 \%$ & $0.43[0.10,0.76]$ & & & $\uparrow$ & \\
\hline \multicolumn{9}{|c|}{$\begin{array}{l}\text { Heterogeneity: } \text { Tau }^{2}=0.04 ; \mathrm{Ch}^{2}=6.78, \mathrm{df}=5(\mathrm{P}=0.24) ; \mathrm{I}^{2}=26 \% \\
\text { Test for overall effect: } \mathrm{Z}=2.55(\mathrm{P}=0.01) \\
\text { Test for subarous differences: } \mathrm{Ch}^{2}=5.40 . \mathrm{df}=1(\mathrm{P}=0.02) . \mathrm{I}^{2}=81.5 \%\end{array}$} & $\begin{array}{c}1 \\
-10\end{array}$ & -5 & $\begin{array}{ll}1 \\
0\end{array}$ & $\frac{1}{10}$ \\
\hline
\end{tabular}

To summarize all the included studies in this review, the stimulation parameters that appear more helpful in cognitive improvement in MCI patients include high frequency (5$20 \mathrm{~Hz}$ ) rTMS applied over the left or bilateral hemisphere (especially DLPFC or PFC) for $\geq 20$ treatment sessions with a stimulation intensity from 80 to $110 \%$ resting MT. Further, rTMS was found to be safe with no serious adverse effects reported.
To minimize potential heterogeneity, subgroup analyses were performed according to the stimulation site, stimulus frequency, and treatment session number. The most common stimulation target for improving cognitive function in patients with $\mathrm{AD}$ was the DLPFC (Cotelli et al., 2011; Liao et al., 2015). In addition to the DLPFC, the anterior temporal lobe and PFC were also targeting for rTMS, and both showed initial promising results (Zhang et al., 2014; Wan et al., 2016). It is widely known that 
the PFC plays a critical role in cognitive functions (Iimori et al., 2018), which are abnormally disturbed in MCI (Sanford, 2017). Further data are required to elucidate the optimal target site of rTMS for MCI. Our findings showed that high-frequency rTMS significantly enhanced global cognitive function and memory, whereas only one trial utilizing low-frequency rTMS (Wan et al., 2016) showed no significant difference in comparison with the control group (medication treatment). Consistent with previous studies, we found that high-frequency rTMS was effective on cognitive function (Nardone et al., 2014; Dong et al., 2018). However, in a single-session study (Turriziani et al., 2012), low-frequency rTMS of the right DLPFC enhanced recognition memory in eight subjects with MCI. Turriziani et al. (2012) speculated that inhibition of the right DLPFC may modulate the activity of this dysfunctional network, restoring an adaptive equilibrium in MCI. All included studies assessing changes in cognition immediately after the intervention suggested that a high number of rTMS treatments ( $\geq 20$ times) produced better global cognitive function and memory effects in MCI. This metaanalysis is consistent with a previous study that suggested that a longer duration of treatment was more effective in patients with AD (Lin et al., 2019; Wang et al., 2020b). However, these results should be interpreted with caution due to the relatively small number of trials, particularly for low-frequency rTMS. Additionally, the GRADE level of evidences for the most of cognitive outcome measures were rated as "moderate"; thus, higher quality studies are required to investigate the use of rTMS for MCI.

Stimulus intensity is determined on the basis of resting MT, which mostly ranges from 80 to $110 \%$ resting MT. Previous studies revealed that the distance between the scalp and motor cortex increases linearly with MT (Herbsman et al., 2009), and the scalp-cortex distance alone may account for $60 \%$ of the variance in measurements of MT (Sabesan et al., 2015). Due to age-related brain atrophy, the scalp-cortex distance in older adults increases, thus the stimulus intensity should be adjusted considering the rate of cortical atrophy. Jorge et al. (2008) noted that the delivery of a high number of pulses was more effective than those who received smaller number of pulses a day in the elderly patients with depression. The appropriate stimulus intensity and number of pulses delivered should be systematically explored among the elderly in future studies (Iriarte and George, 2018). In addition, a wide variety of cognitive measurement tools across studies also contributed to the variation in research results. Because of multifactorial nature of neuropsychological tests, and not easily classified into single domains, thus distinct cognition classification systems have been used to facilitate interpretation. Both executive function and attention are complex and multi-dimensional cognitive constructs, and attention may be considered a specific example of executive function (Yogev-Seligmann et al., 2008; Schmitt et al., 2019). We classified the neuropsychological tests into three cognitive domains, and combined executive function with attention cognitive domains to provide a general framework in this meta-analysis (Karssemeijer et al., 2017).

rTMS is a promising, non-invasive treatment for the improvement of cognitive function in elderly patients with cognitive impairment. Recently, some studies (Ren et al., 2017; Cui et al., 2019) have also combined TMS with neuroimaging and genetic approaches to further understand the potential mechanisms underlying the rTMS effects on cognition. The default mode network (DMN), serum lipid levels (such as cholesterol and triglyceride levels) (Weng et al., 2018; Wang et al., 2020a; Yang et al., 2020), and oxidative stress (such as superoxide dismutase) (Zhu et al., 2019) have been reported to play critical roles in modulating cognitive function in age-related neurodegenerative diseases. Cui et al. (2019) showed that rTMS-induced hypoconnectivity within DMN was associated with clinical cognitive improvements in patients with amnestic MCI. High-frequency rTMS reportedly decreased serum lipid levels (including the total cholesterol and triglyceride) in the healthy older adults (Ren et al., 2017). A review presents that the therapeutic effect of TMS could be mediated at least partly by its effects on antioxidant enzymes (Medina-Fernández et al., 2018). However, MCI is still a heterogeneous clinical construct, typically divided into amnestic or non-amnestic types. Conventional classification system had a limitation, which combined patients with very different cognitive profiles (Edmonds et al., 2016). Therefore, the exploration of cluster analysis may hold great potential in finding robustly replicable subtypes (Qian and Huang, 2019; Freitas, 2020). Future studies combining TMS parameters with successful MCI subtyping and markers in imaging, biochemical, and genetic are useful to achieve more effective disease-modifying therapies (Oulas et al., 2019; Xie et al., 2019).

With respect to treatment acceptance, the present results suggested that rTMS treatment was well-tolerated, and there was no significant difference in dropout rate between the rTMS and control groups. The reason for the loss to followup was primarily unrelated to rTMS treatment, and none of the included trials reported serious adverse effects. On the other hand, the results of this study also suggest that rTMS treatment has better safety compared to control group. Although patients in the rTMS treatment group were found to be more prone to adverse effects than those in the control group, the adverse effects associated with rTMS were rare and mild. The most common were transient headache and dizziness.

\section{Limitations}

A few limitations should be considered when interpreting the findings of the current study. First, the sample sizes of the included studies were small, ranging from 21 to 80 participants, which may limit the statistical power to detect the effects of rTMS on cognitive function in MCI patients. Second, there was considerable heterogeneity in the included studies with respect to the stimulation parameters (frequency, intensity, and pulses). Therefore, the optimal rTMS parameters are unclear. Finally, most of the included trials were conducted in China, only one trial was from Brazil, which may have resulted in a certain degree of language selection bias. In addition, there was another possible selection bias, since only five studies clearly described random sequence generation and 
one study described allocation concealment in detail (Yuan et al., 2020).

\section{Implications}

In conclusion, the present meta-analysis study may suggest a favorable effect of rTMS on cognitive function in patients with MCI. However, there are many parameters that may affect the treatment, such as the intensity of the stimulus, frequency of the stimulus train, the site for stimulation, or even the course of treatment. Further studies should focus on the mechanism and the optimal parameter setting of rTMS, which will be of great importance for the development of this new intervention in clinical practice.

\section{DATA AVAILABILITY STATEMENT}

The original contributions presented in the study are included in the article/Supplementary Materials, further inquiries can be directed to the corresponding author/s.

\section{AUTHOR CONTRIBUTIONS}

$\mathrm{LJ}$ and $\mathrm{CZ}$ were responsible for the literature screening and data extraction. XC, NG, and $\mathrm{YZ}$ were responsible for risk of bias assessment. LJ and $\mathrm{HC}$ were responsible for statistical analysis and writing up the article. JW, ZY, and CL were responsible for

\section{REFERENCES}

Albert, M. S., DeKosky, S. T., Dickson, D., Dubois, B., Feldman, H. H., Fox, N. C., et al. (2011). The diagnosis of mild cognitive impairment due to Alzheimer's disease: recommendations from the National Institute on AgingAlzheimer's Association workgroups on diagnostic guidelines for Alzheimer's disease. Alzheimers. Dement 7, 270-279. doi: 10.1016/j.jalz.2011.03.008

Cheng, C. P. W., Wong, C. S. M., Lee, K. K., Chan, A. P. K., Yeung, J. W. F., and Chan, W. C. (2018). Effects of repetitive transcranial magnetic stimulation on improvement of cognition in elderly patients with cognitive impairment: a systematic review and meta-analysis. Int. J. Geriatr. Psychiatry 33, e1-e13. doi: $10.1002 /$ gps. 4726

Chou, Y. H., Ton That, V., and Sundman, M. (2020). A systematic review and meta-analysis of rTMS effects on cognitive enhancement in mild cognitive impairment and Alzheimer's disease. Neurobiol. Aging 86, 1-10. doi: 10.1016/j.neurobiolaging.2019.08.020

Cotelli, M., Calabria, M., Manenti, R., Rosini, S., Zanetti, O., Cappa, S. F., et al. (2011). Improved language performance in Alzheimer disease following brain stimulation. J. Neurol. Neurosurg. Psychiatr. 82, 794-797. doi: 10.1136/jnnp.2009.197848

Cui, H., Ren, R., Lin, G., Zou, Y., Jiang, L., Wei, Z., et al. (2019). Repetitive transcranial magnetic stimulation induced hypoconnectivity within the default mode network yields cognitive improvements in amnestic mild cognitive impairment: a randomized controlled study. J. Alzheimers. Dis. 69, 1137-1151. doi: 10.3233/JAD-181296

Ding, D., Zhao, Q., Guo, Q., Liang, X., Luo, J., Yu, L., et al. (2016). Progression and predictors of mild cognitive impairment in Chinese elderly: a prospective follow-up in the Shanghai Aging Study. Alzheimers Dement (Amst) 4, 28-36. doi: 10.1016/j.dadm.2016.03.004

Dong, X., Yan, L., Huang, L., Guan, X., Dong, C., Tao, H., et al. (2018). Repetitive transcranial magnetic stimulation for the treatment of Alzheimer's disease: a systematic review and meta-analysis of randomized controlled trials. PLoS ONE 13:e0205704. doi: 10.1371/journal.pone.0205704 planning and guidance on this paper. All authors contributed to the article and approved the submitted version.

\section{FUNDING}

This work was supported by National Key R\&D Program of China (2020YFC2003000 and 2018YFC2001605), National Nature Science Foundation of China (81901400, 81371505, 81971682, 81571756, and 81270023), Science and Technology Commission of Shanghai Municipality (19411968600, 18411952400, 134119a2501, and 13dz2260500), Shanghai Municipal Commission of Health and Family Planning (20174Y0021), Shanghai Municipal Natural Science Foundation (18ZR1432600 and 20ZR1472800), the SHSMU-ION Research Center for Brain Disorders (2017NKX003), Shanghai Municipal Commission of Education-Gaofeng Clinical Medicine Grant Support (20171929), Hundred-Talent Fund from Shanghai Municipal Commission of Health (2018BR17), and the Shanghai Mental Health Center (CRC2017ZD01 and CRC2018DSJ01-5).

\section{SUPPLEMENTARY MATERIAL}

The Supplementary Material for this article can be found online at: https://www.frontiersin.org/articles/10.3389/fnagi. 2020.593000/full\#supplementary-material

Drumond Marra, H. L., Myczkowski, M. L., Maia Memória, C., Arnaut, D., Leite Ribeiro, P., Sardinha Mansur, C. G., et al. (2015). Transcranial magnetic stimulation to address mild cognitive impairment in the elderly: a randomized controlled study. Behav. Neurol. 2015:287843. doi: 10.1155/2015/287843

Edmonds, E. C., Eppig, J., Bondi, M. W., Leyden, K. M., Goodwin, B., and Delano-Wood, L., et al. (2016). Heterogeneous cortical atrophy patterns in MCI not captured by conventional diagnostic criteria. Neurology 87, 2108-2116. doi: 10.1212/WNL.00000000000 03326

Feng, W., Wang, D., Tang, L., Cheng, Y., Wang, G., Hu, G., et al. (2018). Effects of different cognitive trainings on amnestic mild cognitive impairment in the elderly: a one-year longitudinal functional magnetic resonance imaging (MRI) study. Med. Sci. Monit. 24, 5517-5527. doi: 10.12659/MSM.908315

Folstein, M. F., Folstein, S. E., and McHugh, P. R. (1975). "Mini-mental state": a practical method for grading the cognitive state of patients for the clinician. $J$. Psychiatr. Res. 12, 189-198. doi: 10.1016/0022-3956(75)90026-6

Freitas, A. A. (2020). Investigating the role of Simpson's paradox in the analysis of top-ranked features in high-dimensional bioinformatics datasets. Brief Bioinform 21, 421-428. doi: 10.1093/bib/bby126

Guo, Q., Li, C., and Wang, J. (2017). Updated review on the clinical use of repetitive transcranial magnetic stimulation in psychiatric disorders. Neurosci. Bull 33, 747-756. doi: 10.1007/s12264-017-0185-3

Guyatt, G. H., Oxman, A. D., Vist, G. E., Kunz, R., Falck-Ytter, Y., AlonsoCoello, P., et al. (2008). GRADE: an emerging consensus on rating quality of evidence and strength of recommendations. BMJ 336, 924-926. doi: 10.1136/bmj.39489.470347.AD

Han, K., Yu, L., Wang, L., Li, L., Liu, K., Xu, S., et al. (2013). The case-control study of the effect of repetitive transcranial magnetic stimulation on elderly mild cognitive impairment patients. J. Clin. Psychiatry 23, 156-159.

Herbsman, T., Forster, L., Molnar, C., Dougherty, R., Christie, D., Koola, J., et al. (2009). Motor threshold in transcranial magnetic stimulation: the impact of white matter fiber orientation and skull-to-cortex distance. Hum. Brain Mapp. 30, 2044-2055. doi: 10.1002/hbm.20649 
Higgins, J. P. T., and Green, S. (2011). Cochrane Handbook for Systematic Reviews of Interventions Version 5.1.0 updated March 2011. The Cochrane Collaboration. Available online at: www.cochrane-handbook.org

Hsu, W. Y., Ku, Y., Zanto, T. P., and Gazzaley, A. (2015). Effects of noninvasive brain stimulation on cognitive function in healthy aging and Alzheimer's disease: a systematic review and meta-analysis. Neurobiol. Aging 36, 2348-2359. doi: 10.1016/j.neurobiolaging.2015.04.016

Iimori, T., Nakajima, S., Miyazaki, T., Tarumi, R., Ogyu, K., Wada, M., et al. (2018). Effectiveness of the prefrontal repetitive transcranial magnetic stimulation on cognitive profiles in depression, schizophrenia, and Alzheimer's disease: a systematic review. Prog. Neuropsychopharmacol. Biol. Psychiatry 88, 31-40. doi: 10.1016/j.pnpbp.2018.06.014

Iriarte, I. G., and George, M. S. (2018). Transcranial Magnetic Stimulation (TMS) in the Elderly. Curr. Psychiatry Rep. 20:6. doi: 10.1007/s11920-018-0866-2

Jorge, R. E., Moser, D. J., Acion, L., and Robinson, R. G. (2008). Treatment of vascular depression using repetitive transcranial magnetic stimulation. Arch. Gen. Psychiatry 65, 268-276. doi: 10.1001/archgenpsychiatry.2007.45

Karssemeijer, E. G. A., Aaronson, J. A., Bossers, W. J., Smits, T., Olde Rikkert, M. G. M., and Kessels, R. P. C. (2017). Positive effects of combined cognitive and physical exercise training on cognitive function in older adults with mild cognitive impairment or dementia: a meta-analysis. Ageing Res. Rev. 40, 75-83. doi: 10.1016/j.arr.2017.09.003

Kasper, S., Bancher, C., Eckert, A., Förstl, H., Frölich, L., Hort, J., et al. (2020). Management of mild cognitive impairment (MCI): the need for national and international guidelines. World J. Biol. Psychiatry 5, 1-16. doi: 10.1080/15622975.2019.1696473

LeWitt, P. A., Kymes, S., and Hauser, R. A. (2020). Parkinson disease and orthostatic hypotension in the elderly: recognition and management of risk factors for falls. Aging Dis. 11, 679-691. doi: 10.14336/AD.2019.0805

Li, R., Huang, X., Liang, X., Su, M., Lai, K. P., and Chen, J. (2020). Integrated omics analysis reveals the alteration of gut microbe-metabolites in obese adults. Brief Bioinform. doi: 10.1093/bib/bbaa165. [Epub ahead of print].

Liao, X., Li, G., Wang, A., Liu, T., Feng, S., Guo, Z., et al. (2015). Repetitive transcranial magnetic stimulation as an alternative therapy for cognitive impairment in Alzheimer's disease: a meta-analysis. J. Alzheimers. Dis. 48, 463-472. doi: 10.3233/JAD-150346

Liberati, A., Altman, D. G., Tetzlaff, J., Mulrow, C., Gøtzsche, P. C., Ioannidis, J. P., et al. (2009). The PRISMA statement for reporting systematic reviews and meta-analyses of studies that evaluate healthcare interventions: explanation and elaboration. BMJ 339:b2700. doi: 10.1136/bmj.b2700

Lin, Y., Jiang, W. J., Shan, P. Y., Lu, M., Wang, T., Li, R. H., et al. (2019). The role of repetitive transcranial magnetic stimulation (rTMS) in the treatment of cognitive impairment in patients with Alzheimer's disease: a systematic review and meta-analysis. J. Neurol. Sci. 398, 184-191. doi: 10.1016/j.jns.2019.01.038

Long, S., Wang, X., Luo, C., Liu, X., Yang, F., He, S., et al. (2016). Impacts of repetitive transcranial magnetic stimulation on the brain network regulation and cognitive function of patients with amnesiac mild cognitive impairment. $J$. Epileptol. Electroneurophysiol (Chin) 25, 6-11.

Ma, L., and Chan, P. (2020). Understanding the physiological links between physical frailty and cognitive decline. Aging Dis. 11, 405-418. doi: 10.14336/AD.2019.0521

Medina-Fernández, F. J., Escribano, B. M., Padilla-Del-Campo, C., Drucker-Colín, R., Pascual-Leone, Á., and Túnez, I. (2018). Transcranial magnetic stimulation as an antioxidant. Free Radic. Res. 52, 381-389. doi: 10.1080/10715762.2018. 1434313

Najib, U., Bashir, S., Edwards, D., Rotenberg, A., and Pascual-Leone, A. (2011). Transcranial brain stimulation: clinical applications and future directions. Neurosurg. Clin. N. Am. 22, 233-251. doi: 10.1016/j.nec.2011. 01.002

Nardone, R., Tezzon, F., Höller, Y., Golaszewski, S., Trinka, E., and Brigo, F. (2014). Transcranial magnetic stimulation (TMS)/repetitive TMS in mild cognitive impairment and Alzheimer's disease. Acta Neurol. Scand. 129, 351-366. doi: 10.1111/ane.12223

Oulas, A., Minadakis, G., Zachariou, M., Sokratous, K., Bourdakou, M. M., and Spyrou, G. M. (2019). Systems Bioinformatics: increasing precision of computational diagnostics and therapeutics through networkbased approaches. Brief Bioinform. 20, 806-824. doi: 10.1093/bib/ bbx151
Petersen, R. C., Roberts, R. O., Knopman, D. S., Boeve, B. F., Geda, Y. E., Ivnik, R. J., et al. (2009). Mild cognitive impairment: ten years later. Arch. Neurol. 66, 1447-1455. doi: 10.1001/archneurol.2009.266

Petersen, R. C., Smith, G. E., Waring, S. C., Ivnik, R. J., Tangalos, E. G., and Kokmen, E. (1999). Mild cognitive impairment: clinical characterization and outcome. Arch. Neurol. 56, 303-308. doi: 10.1001/archneur. 56.3.303

Qian, E., and Huang, Y. (2019). Subtyping of Parkinson's disease - where are we up to? Aging Dis. 10, 1130-1139. doi: 10.14336/AD.2019.0112

Ren, W., Ma, J., Li, J., Zhang, Z., and Wang, M. (2017). Repetitive transcranial magnetic stimulation (rTMS) modulates lipid metabolism in aging adults. Front. Aging Neurosci. 9:334. doi: 10.3389/fnagi.2017.00334

Rossini, P. M., Barker, A. T., Berardelli, A., Caramia, M. D., Caruso, G., Cracco, R. Q., et al. (1994). Non-invasive electrical and magnetic stimulation of the brain, spinal cord and roots: basic principles and procedures for routine clinical application. Report of an IFCN committee. Electroencephalogr. Clin. Neurophysiol. 91, 79-92. doi: 10.1016/0013-4694(94)90029-9

Sabesan, P., Lankappa, S., Khalifa, N., Krishnan, V., Gandhi, R., and Palaniyappan, L. (2015). Transcranial magnetic stimulation for geriatric depression: promises and pitfalls. World J. Psychiatry 5, 170-181. doi: 10.5498/wjp.v5.i2.170

Sanford, A. M. (2017). Mild cognitive impairment. Clin. Geriatr. Med. 33, 325-337. doi: 10.1016/j.cger.2017.02.005

Schmitt, L. M., Shaffer, R. C., Hessl, D., and Erickson, C. (2019). Executive function in fragile $\mathrm{X}$ syndrome: a systematic review. Brain Sci. 9:15. doi: 10.3390/brainsci9010015

Sun, R., and Ma, Y. (2015). Effect of repetitive transcranial magnetic stimulation combined with cognitive training on mild cognitive impairment. Chin. J. Rehabil. 30, 355-357. doi: 10.3870/zgkf.2015.05.010

Turriziani, P., Smirni, D., Zappala, G., Mangano, G. R., Oliveri, M., and Cipolotti, L. (2012). Enhancing memory performance with rTMS in healthy subjects and individuals with mild cognitive impairment: the role of the right dorsolateral prefrontal cortex. Front. Hum. Neurosci. 6:62. doi: 10.3389/fnhum.2012.00062

Wan, J., Xu, M., Sui, R., and Tao, H. (2016). rTMS and computerbased cognitive training improve cognitive function in patients with mild cognitive impairment. Chin. J. Geriatr. Heart Brain Vessel Dis. 18, 356-362. doi: 10.3969/jissn.1009-0126.2016.04.006

Wang, M., Pan, X., Deng, W., Yang, Y., Chen, S., Hu, R., et al. (2015). Efficacy of repetitive transcranial magnetic stimulation on patients with mild cognitive impairment: a systematic review and meta-analysis. Chin. J. Evid-based Med. 15, 1393-1400. doi: 10.7507/1672-2531.20150228

Wang, T., Yuan, F., Chen, Z., Zhu, S., Chang, Z., Yang, W., et al. (2020a). Vascular, inflammatory and metabolic risk factors in relation to dementia in Parkinson's disease patients with type 2 diabetes mellitus. Aging (Albany NY) 12, 15682-15704. doi: 10.18632/aging. 103776

Wang, X., Mao, Z., Ling, Z., and Yu, X. (2020b). Repetitive transcranial magnetic stimulation for cognitive impairment in Alzheimer's disease: a meta-analysis of randomized controlled trials. J. Neurol. 267, 791-801. doi: 10.1007/s00415-019-09644-y

Wei, Y., Zhu, J., Pan, S., Su, H., Li, H., and Wang, J. (2017). Meta-analysis of the efficacy and safety of Repetitive Transcranial Magnetic Stimulation (rTMS) in the treatment of depression. Shanghai Arch. Psychiatry 29, 328-342. doi: 10.11919/j.issn.1002-0829.217106

Wen, X., Cao, X., Qiu, G., Dou, Z., and Chen, S. (2018). Effects of repetitive transcranial magnetic stimulation on amnestic mild cognitive impairment. Chin. J. Gerontol. 38, 1662-1663.

Weng, R., Wei, X., Yu, B., Zhu, S., Yang, X., Xie, F., Zhang M., et al. (2018). Combined measurement of plasma cystatin $\mathrm{c}$ and low-density lipoprotein cholesterol: a valuable tool for evaluating progressive supranuclear palsy. Parkinsonism Relat. Disord. 52, 37-42. doi: 10.1016/j.parkreldis.2018.03.014

Xie, F., Gao, X., Yang, W., Chang, Z., Yang, X., et al. (2019). Advances in the research of risk factors and prodromal biomarkers of parkinson's disease. ACS Chem. Neurosci. 10, 973-990. doi: 10.1021/acschemneuro.8b00520

Xu, J., Fu, X., Pan, M., Zhou, X., Chen, Z., Wang, D., Zhang X., et al. (2019a). Mitochondrial creatine kinase is decreased in the serum of idiopathic parkinson's disease patients. Aging Dis. 10, 601-610. doi: 10.14336/AD.2018.0615

Xu, Y., Qiu, Z., Zhu, J., Liu, J., Wu, J., Tao, J., et al. (2019b). The modulation effect of non-invasive brain stimulation on cognitive function in patients with mild 
cognitive impairment: a systematic review and meta-analysis of randomized controlled trials. BMC Neurosci. 20:2. doi: 10.1186/s12868-018-0484-2

Yang, L., Han, K., Wang, X., An, C., Xu, S., Song, M., et al. (2014). Preliminary study of high-frequency repetitive transcranial magnetic stimulation in treatment of mild cognitive impairment. Chin. J. Psychiatry 47, 227-231. doi: 10.3760/cma.j.issn.1006-7884.2014.04.008

Yang, W., Chang, Z., Que, R., Weng, G., Deng, B., Wang, T., et al. (2020). Contra-directional expression of plasma superoxide dismutase with lipoprotein cholesterol and high-sensitivity C-reactive protein as important markers of parkinson's disease severity. Front. Aging Neurosci. 12:53. doi: 10.3389/fnagi.2020.00053

Yogev-Seligmann, G., Hausdorff, J. M., and Giladi, N. (2008). The role of executive function and attention in gait. Mov. Disord. 23, 329-342. doi: $10.1002 / \mathrm{mds} .21720$

Yuan, F., Guo, X., Wei, X., Xie, F., Zheng, J., Huang, Y., et al. (2020). Lee Silverman Voice Treatment for dysarthria in patients with Parkinson's disease: a systematic review and meta-analysis. Eur. J. Neurol. 27, 1957-1970. doi: 10.1111/ene.14399

Zhang, L., Yuan, L., and Wang, Y. (2014). Effects of repetitive transcranial magnetic stimulation on cognitive function in patients with mild cognitive impairment. J. Clin. Neurol. 27, 203-206.
Zhu, S., Wei, X., Yang, X., Huang, Z., Chang, Z., Xie, F., et al. (2019). Plasma lipoprotein-associated phospholipase A2 and superoxide dismutase are independent predicators of cognitive impairment in cerebral small vessel disease patients: diagnosis and assessment. Aging Dis. 10, 834-846. doi: 10.14336/AD.2019.0304

Zou, J., Chen, Z., Liang, C., Fu, Y., Wei, X., Lu, J., et al. (2018). Trefoil factor 3, cholinesterase and homocysteine: potential predictors for Parkinson's disease dementia and vascular parkinsonism dementia in advanced stage. Aging Dis. 9, 51-65. doi: 10.14336/AD.2017.0416

Conflict of Interest: The authors declare that the research was conducted in the absence of any commercial or financial relationships that could be construed as a potential conflict of interest.

Copyright $\odot 2021$ Jiang, Cui, Zhang, Cao, Gu, Zhu, Wang, Yang and Li. This is an open-access article distributed under the terms of the Creative Commons Attribution License (CC BY). The use, distribution or reproduction in other forums is permitted, provided the original author(s) and the copyright owner(s) are credited and that the original publication in this journal is cited, in accordance with accepted academic practice. No use, distribution or reproduction is permitted which does not comply with these terms. 